\title{
Engaging policy-makers, health system managers, and policy analysts in the knowledge synthesis process: a scoping review
}

Andrea C. Tricco ${ }^{1,2^{*}}$ (D), Wasifa Zarin ${ }^{1}$, Patricia Rios ${ }^{1}$, Vera Nincic ${ }^{1}$, Paul A. Khan ${ }^{1}$, Marco Ghassemi ${ }^{1}$, Sanober Diaz ${ }^{1}$, ${\text { Ba' }{ }^{\prime} \text { Pham }}^{1}$, Sharon E. Straus ${ }^{3}$ and Etienne V. Langlois ${ }^{4}$

\begin{abstract}
Background: It is unclear how to engage a wide range of knowledge users in research. We aimed to map the evidence on engaging knowledge users with an emphasis on policy-makers, health system managers, and policy analysts in the knowledge synthesis process through a scoping review.
\end{abstract}

Methods: We used the Joanna Briggs Institute guidance for scoping reviews. Nine electronic databases (e.g., MEDLINE) , two grey literature sources (e.g., OpenSIGLE), and reference lists of relevant systematic reviews were searched from 1996 to August 2016. We included any type of study describing strategies, barriers and facilitators, or assessing the impact of engaging policy-makers, health system managers, and policy analysts in the knowledge synthesis process. Screening and data abstraction were conducted by two reviewers independently with a third reviewer resolving discrepancies. Frequency and thematic analyses were conducted.

Results: After screening 8395 titles and abstracts followed by 394 full-texts, 84 unique documents and 7 companion reports fulfilled our eligibility criteria. All 84 documents were published in the last 10 years, and half were prepared in North America. The most common type of knowledge synthesis with knowledge user engagement was a systematic review (36\%). The knowledge synthesis most commonly addressed an issue at the level of national healthcare system (48\%) and focused on health services delivery (17\%) in high-income countries (86\%).

Policy-makers were the most common (64\%) knowledge users, followed by healthcare professionals (49\%) and government agencies as well as patients and caregivers (34\%). Knowledge users were engaged in conceptualization and design (49\%), literature search and data collection (52\%), data synthesis and interpretation (71\%), and knowledge dissemination and application (44\%). Knowledge users were most commonly engaged as key informants through meetings and workshops as well as surveys, focus groups, and interviews either in-person or by telephone and emails. Knowledge user content expertise/awareness was a common facilitator (18\%), while lack of time or opportunity to participate was a common barrier (12\%).

Conclusions: Knowledge users were most commonly engaged during the data synthesis and interpretation phases of the knowledge synthesis conduct. Researchers should document and evaluate knowledge user engagement in knowledge synthesis.

(Continued on next page)

\footnotetext{
*Correspondence: triccoa@smh.ca

'Li Ka Shing Knowledge Institute of St. Michael's Hospital, 209 Victoria Street,

Toronto, Ontario M5B 1T8, Canada

${ }^{2}$ Epidemiology Division, Dalla Lana School of Public Health, University of

Toronto, 6th Floor, 155 College St, Toronto, Ontario M5T 3M7, Canada

Full list of author information is available at the end of the article
} 
(Continued from previous page)

Registration details: Open Science Framework (https://osf.io/4dy53/).

Keywords: Engagement, Knowledge user, Stakeholder, Knowledge translation, Health policy, Policy-relevant, Health system, Policy-maker, Knowledge synthesis

\section{Background}

An estimated $85 \%$ of investment in health and biomedical research is wasted every year due to redundancies, failure to establish priorities based on needs of stakeholders (particularly end-users of knowledge), poorly designed research methods, and incomplete reporting of study results, leading to billions of dollars lost globally [1-3]. Stakeholders include those who are affected by, have an interest or stake in research [4], while knowledge users are subgroup of stakeholders who are likely to use research findings to make informed decisions about health systems and practices [5]. Knowledge users include but are not limited to patients and their informal caregivers or surrogate decision-makers (e.g., family, friends), healthcare providers (e.g., physicians, occupational therapists), policy-makers (e.g., Minister of Health, health officer), health system managers (e.g., hospital administrators, health unit managers), and policy analysts.

The overarching goal of knowledge user engagement in health research is to co-produce knowledge that is relevant and useful to those making real-world health decisions [6]. Early engagement of knowledge users in the research process may help establish research priorities and increase relevance of findings $[7,8]$. To facilitate the use of research in decision-making, health systems and research funders are encouraging the engagement of knowledge users and other stakeholders in research [9].

Knowledge synthesis, such as a systematic review or a scoping review, is particularly useful for decision-makers because these research products provide a summary of the expansive evidence on a particular topic to inform decisions based on the totality of evidence [10, 11]. Co-production of evidence whereby researchers and knowledge users work together to conduct research increases the policy-relevance of research questions and fosters integration of findings into policy and practice [12-14]. However, the opportunities and approaches to engaging a wide range of knowledge users remain largely unexplored. Evidence is required to guide the process of engaging knowledge users in knowledge synthesis to identify engagement approaches that are effective, efficient, and meaningful. In addition, co-production of research by researchers and knowledge users requires additional time and funding, and it is imperative that the limited resources available for health research are used appropriately.

We undertook a scoping review to map the literature on engaging knowledge users in the knowledge synthesis process. Engagement of policy-makers, policy analysts, and health system managers were of particular interest, as these knowledge users are increasingly commissioning knowledge synthesis research products to meet their decision-making needs. The research questions (RQs) for our scoping review are provided below and outlined in our published protocol [15]:

(RQ1) In what context were policy-makers, health system managers, and policy analysts engaged (e.g., health system setting, high-income countries (HICs) versus low and middle-income countries (LMICs))?

(RQ2) What strategies exist to engage policy-makers, health system managers, and policy analysts in the knowledge synthesis process?

(RQ3) In studies describing strategies for engaging policy-makers, health system managers, and policy analysts, what outcomes do they measure to evaluate engagement mechanisms (e.g., attitudes, beliefs, knowledge) and what are the results (e.g. benefits, unintended consequences)?

(RQ4) What are the barriers and facilitators in engaging policy-makers, health system managers, and policy analysts in the knowledge synthesis process?

\section{Methods}

\section{Commissioning agency}

As part of a project to strengthen research capacity in LMICs, we were commissioned to conduct this scoping review by the Alliance for Health Policy and Systems Research (hereafter the Alliance), an international partnership hosted by the World Health Organization (WHO). We engaged with members of the Alliance throughout the review conduct.

\section{Study design}

We selected the scoping review method [16] because we were interested in mapping the concepts relevant to engaging knowledge users in knowledge synthesis $[16,17]$. The scoping review methodology is particularly useful when exploring an emerging and diverse knowledge-base, which makes the method well-matched to our RQs.

\section{Protocol}

We drafted a scoping review protocol following the methods outlined by the Joanna Briggs Institute Methods Manual for scoping reviews [18] and reported findings using the elements provided in the Preferred Reporting Items for Systematic Reviews and Meta-analysis for 
Protocols (PRISMA-P) [19]. Our protocol was revised by the research team, registered with the Open Science Framework [20], and published in BMJ Open [15]. Since our full methods are available in our protocol, they are outlined briefly below.

\section{Eligibility criteria}

Our eligibility criteria were conceptualized using the Population, Intervention, Comparator, Outcome, and Study design components [21], as follows:

\section{Population}

At minimum, the paper must mention at least one of the three knowledge user types specified in our RQs, which included policy-makers, policy analysts, and health system managers. Policy-makers are individuals at some level of government or decision-making institution, including but not limited to international organizations, nongovernmental agencies or professional associations, who have responsibility for making recommendations to others [22]. Policy analysts are individuals at some level of government or decision-making institution, including but not limited to international organizations, non-governmental agencies or professional associations, responsible for analyzing data and informing decisions and recommendations [22]. Health system managers are individuals in a managerial or supervisory role in a health system with management or supervisory mandates, including implementers and public health officials [22].

\section{Intervention}

Papers that described any engagement strategy for policymakers, health system managers, and policy analysts in the knowledge synthesis process were included. Engagement can be defined as "an iterative process of actively soliciting the knowledge, experience, judgment and values of individuals selected to represent a broad range of direct interests in a particular issue, for the dual purposes of: creating a shared understanding [and] making relevant, transparent and effective decisions" [8]. This scoping review limits knowledge user engagement to those opportunities that allow a meaningful interaction of the knowledge users in the research process from conception to design and completion and/or interpretation and uptake of results.

\section{Comparators}

Papers with or without a comparator group were eligible for inclusion.

\section{Outcomes}

Outcomes of interest were strategies, barriers, facilitators, and contextual factors for engaging health policymakers, health system managers, or policy analysts in the conduct and use of knowledge synthesis. We also explored whether engagement strategies were evaluated regarding researcher and knowledge user attitudes, beliefs and knowledge of engagement as well as impact and effectiveness of engagement.

\section{Study designs}

We included any type of study design (e.g., qualitative or quantitative methods).

\section{Time periods}

To increase feasibility and timeliness of review completion, we restricted inclusion of the literature to the past 20 years.

\section{Setting}

All settings were eligible for inclusion.

\section{Other}

To increase feasibility and timeliness of review completion, only papers written in English were included.

Our full list of eligibility criteria can be found in Additional file 1: Appendix 1.

\section{Information sources and search strategy}

The following electronic databases were searched by an experienced librarian (Dr. Jessie McGowan) from 1996 to August 15 2016: MEDLINE, Embase, ERIC, PsycINFO, Joanna Briggs, The Cochrane Library, EBM Reviews, The Campbell Library, and Social Work abstracts. The MEDLINE search strategy was peer-reviewed using the PRESS Statement [23] by a second librarian (Dr. Elise Cogo) and has been published in our protocol [15]. The main literature search was supplemented through searching GreyNet International [24] and OpenSIGLE [25] to locate unpublished (or grey) literature, such as conference abstracts and dissertations. All literature searches and full-text retrievals were executed by an experienced library technician (Ms. Alissa Epworth) and managed using Endnote [26]. Additionally, references from relevant review articles were scanned, and experts in the field were identified and contacted via email by the Alliance to identify additional sources of evidence.

\section{Study selection process}

Literature search results were screened using our online Synthesi.SR software [27]. For level 1 screening of titles and abstracts, 3 pilot-tests were conducted on a total of 125 records. Once $80 \%$ agreement was achieved, pairs of reviewers (BP, MG, $\mathrm{PR}, \mathrm{PK}, \mathrm{SD}, \mathrm{VN}$, and $\mathrm{WZ}$ ) independently screened remaining titles and abstracts. There were 403 (5\%) discrepancies at level 1 screening, which were resolved by a third reviewer (WZ). For level 2 screening of potentially relevant full-text articles, 2 pilot-tests were conducted. When 70\% agreement was achieved, pairs of reviewers (MG, $\mathrm{PR}, \mathrm{PK}, \mathrm{SD}, \mathrm{VN}$, and WZ) independently screened the full- 
text articles. There were $62(18 \%)$ discrepancies at level 2 screening, which were resolved by a third reviewer (WZ).

\section{Data items and data abstraction process}

We abstracted data on article characteristics (e.g., country of origin, funder), engagement characteristics and contextual factors (e.g., type of knowledge user, country income level [28], type of engagement activity, frequency and intensity of engagement, use of a framework [29] to inform the intervention), barriers and facilitators to engagement, and results of any formal assessment of engagement (e.g., attitudes, beliefs, knowledge, benefits, unintended consequences).

Data abstraction was conducted using a standardized Excel form that was developed $a$ priori and pilot-tested on a sample of 5 included papers. After the team conducted 2 pilot-tests, data was abstracted by one reviewer and verified by another (MG, PK, SD, VN). Two experienced reviewers then quality checked the data for consistency and accuracy (WZ or PR).

\section{Risk of bias assessment}

We did not conduct risk of bias assessment, which is consistent with the Joanna Briggs Institute Scoping
Review Methods Manual [18] and scoping reviews on health-related topics [17].

\section{Synthesis of results}

Results were synthesized using frequencies and thematic analysis [30]. Thematic analysis of open-text data was performed by one reviewer and verified by a second reviewer (PR, WZ). We used previously established nomenclature in our thematic analysis of barriers and facilitators to engaging knowledge users in health research [31, 32]. Engagement was coded and defined based on the framework established by Keown et al. [33]. Metaanalysis was not performed.

\section{Results}

Literature search

After screening 8395 titles and abstracts and 394 fulltext documents, 84 unique documents and 7 companion reports [34-40] (i.e., follow-up reports to the main documents included in our review) fulfilled our eligibility criteria (Fig. 1). The full citations can be found in Additional file 1: Appendix 2. Four documents were excluded because they were written in languages other than English, and 79 were excluded because they were
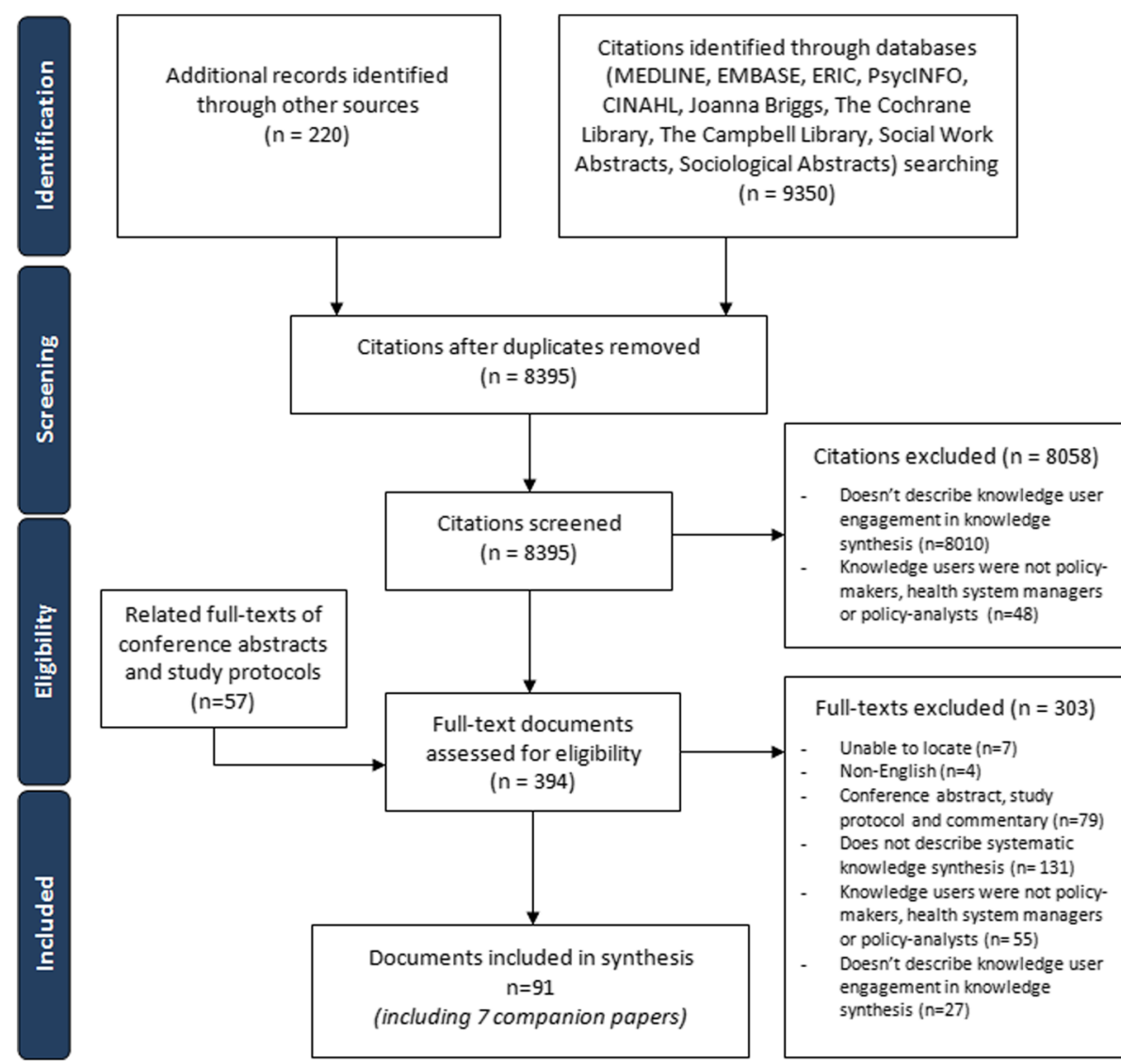
Citations excluded $(\mathrm{n}=8058$ )
Doesn't describe knowledge user engagement in knowledge synthesis $(n=8010)$
Knowledge users were not policy- makers, health system managers or policy-analysts ( $n=48$ )

Fig. 1. PRISMA flow diagram 
conference abstracts, commentaries, or protocols that did not include relevant data. Six documents were identified through contacting experts in the field [11, 18, 2427]. Six were unpublished reports, which were identified through our literature searches as well as through expert contact [41-46]. No relevant documents were identified through scanning reference lists.

\section{Characteristics of included documents $(n=84)$}

All documents were published in the last 10 years (Table 1) and originated predominantly in Canada (37\%), USA (17\%), UK (18\%), and Australia (13\%) (Fig. 2). The funding source was mainly public (79\%), and Health Care Sciences \& Services was the most common publishing journal discipline (31\%). The types of documents were classified as application papers (87\%) which are knowledge synthesis papers that described knowledge user engagement in the conduct of their research, descriptive papers (10\%) which provided details of knowledge user engagement strategies developed by a research center or program, and methodology papers (4\%) that studied knowledge user engagement in the knowledge synthesis process. The most common types of knowledge synthesis with knowledge user engagement were: systematic review (36\%), literature review with a systematic literature search (19\%), scoping review (14\%), rapid review (12\%), and realist review (6\%) (Table 1).

\section{RQ1: Contextual factors of included documents $(n=84)$}

The research most commonly addressed an issue at the level of the national healthcare systems (48\%), followed by applied research settings (19\%), and local healthcare systems (6\%; Table 2). The knowledge synthesis product most commonly focused on health services delivery (17\%), followed by knowledge translation (16\%), and public health (10\%). Most of the documents were produced in the context of high-income countries (86\%), while $12 \%$ of the documents were in the context of lowand middle-income countries (see Table 3 for more details on LMICs).

\section{RQ2: Methodology documents $(n=3)$}

One methodology document conducted 18 key informant interviews with policy-makers and systematic review producers to identify institutional mechanisms to increase demand for and facilitate conduct of policyrelevant systematic reviews [47]. The authors proposed four models for achieving policy-relevant systematic reviews with an emphasis on policy-maker engagement based on knowledge user needs and timelines as well as complexity of the research question. The authors concluded that early engagement with managers and policymakers can improve clarity and consensus of definitions and maximize relevance of systematic reviews.
Another methodology document conducted a systematic literature search of stakeholder engagement and 13 key informant interviews on prioritizing research [48]. The authors included 56 papers that used mixed qualitative/quantitative approaches to engaging stakeholders using in-person, online, or teleconference modalities. Prioritization of research was often achieved using structured ranking or Delphi methods. Ten factors for successful engagement were recommended and are outlined in Table 4.

A third methodology document examined the benefits of engaging a range of stakeholders in systematic reviews through a review of 24 papers and 34 key informant interviews [46]. The authors noted that although a number of benefits and challenges to engaging stakeholders were identified, none of the studies formally evaluated engagement.

\section{RQ2: Descriptive documents $(n=8)$}

The eight descriptive documents [33, 49-54] described engagement approaches used for the following: Healthcare Improvement Scotland [53], Greater London Authority (UK) [55], Agency for Healthcare Research and Quality (US) [49], Samueli Institute (US) [51], Institute for Work and Health (Canada), Ottawa Hospital Research Institute (Canada) [52], and various funding and governmental agencies within Canada [33, 50, 54]. Most of the approaches involved consultations. One paper described [33] engagement of knowledge users as part of the review team. Further results can be found in Table 5 .

\section{RQ2: Application papers $(n=73)$}

Seventy-three knowledge synthesis documents reported knowledge user engagement in the research process. Policy-makers were the most common (64\%) type of knowledge users to be engaged in the knowledge synthesis process, followed by healthcare professionals and organizations (49\%), and government agencies as well as patient organizations and caregivers (34\%; Fig. 3).

The points of engagement in the knowledge synthesis process occurred at the onset of the review to conceptualize and plan the research (49\%), where knowledge users were engaged to either select the research topic or refine research questions (40\%), develop the study proposal or protocol $(29 \%)$ or define study selection criteria (27\%) (Fig. 4). Knowledge users were also involved at the literature search or data collection phase (52\%) to either assist with the literature search (26\%), help with study selection (8\%), provide input on the data collection form (18\%), help with data collection $(5 \%)$ or provide experiential data to supplement the data obtained from the literature searches (32\%). At the data synthesis and interpretation stage (71\%), knowledge users informed data analysis (32\%) or helped interpret the results (66\%). 
Table 1 Document characteristics

\begin{tabular}{|c|c|c|}
\hline Document characteristics $(n=84)$ & & Count (\%) \\
\hline \multirow[t]{4}{*}{ Year of publication } & $2005-2007$ & $6(7.1 \%)$ \\
\hline & $2008-2010$ & $16(19.0 \%)$ \\
\hline & $2011-2013$ & $30(35.7 \%)$ \\
\hline & $2014-2016$ & $32(38.1 \%)$ \\
\hline \multirow[t]{5}{*}{ Geographic region } & Africa & $4(4.8 \%)$ \\
\hline & Asia & $4(4.8 \%)$ \\
\hline & Australia \& New Zealand & $11(13.1 \%)$ \\
\hline & Europe & $20(23.8 \%)$ \\
\hline & North America & $45(53.6 \%)$ \\
\hline \multirow[t]{4}{*}{ Funding source type } & Industry-sponsored & $2(2.4 \%)$ \\
\hline & Non-sponsored & $3(3.6 \%)$ \\
\hline & Not reported & $13(15.5 \%)$ \\
\hline & Public-sponsored & $66(78.6 \%)$ \\
\hline \multirow[t]{7}{*}{ Journal discipline } & General \& Internal Medicine & $4(4.8 \%)$ \\
\hline & Not applicable (reports) & $6(7.1 \%)$ \\
\hline & Medicine, General \& Internal & $6(7.1 \%)$ \\
\hline & Health Policy \& Services & 7 (8.3\%) \\
\hline & Public, Environmental \& Occupational Health & $14(16.7 \%)$ \\
\hline & Other & $21(25.0 \%)$ \\
\hline & Health Care Sciences \& Services & $26(31.0 \%)$ \\
\hline \multirow[t]{13}{*}{ Knowledge synthesis method } & Qualitative review & $1(1.2 \%)$ \\
\hline & Critical Interpretive Synthesis & $1(1.2 \%)$ \\
\hline & Mixed-method review & $1(1.2 \%)$ \\
\hline & Health Technology Assessment & $1(1.2 \%)$ \\
\hline & Scoping Review \& Systematic Review & $1(1.2 \%)$ \\
\hline & Horizontal scan & $1(1.2 \%)$ \\
\hline & Rapid Realist Review & $2(2.4 \%)$ \\
\hline & Overview of Reviews & $3(3.6 \%)$ \\
\hline & Realist Review & $5(6.0 \%)$ \\
\hline & Rapid Review & $10(11.9 \%)$ \\
\hline & Scoping Review & $12(14.3 \%)$ \\
\hline & Literature review & $16(19.0 \%)$ \\
\hline & Systematic review & $30(35.7 \%)$ \\
\hline \multirow[t]{3}{*}{ Article type } & Methodology paper & $3(3.6 \%)$ \\
\hline & Descriptive paper & $8(9.5 \%)$ \\
\hline & Application paper & $73(86.9 \%)$ \\
\hline
\end{tabular}

During the knowledge dissemination and application phase (44\%), knowledge users assisted with the report writing (10\%), reviewed and provided feedback on the draft report (18\%), helped develop key messages (4\%), developed practice or policy recommendations (15\%), or established the future research agenda (4\%).

Knowledge users were most commonly engaged as key informants across the four stages of the knowledge synthesis process (Fig. 5). Other roles included advisors (i.e., knowledge users provide high-level recommendations and advice on the design and method and is typically engaged at various stages of a review), expert panel (i.e., knowledge users provide specialized input/opinion on the topic and is typically engaged at a specific stage of a review), steering group (i.e., knowledge users provide strategic decisions on the direction of the research project and is consulted at various stages of a review), or as a team member (i.e., knowledge user is included as part of the review team). 


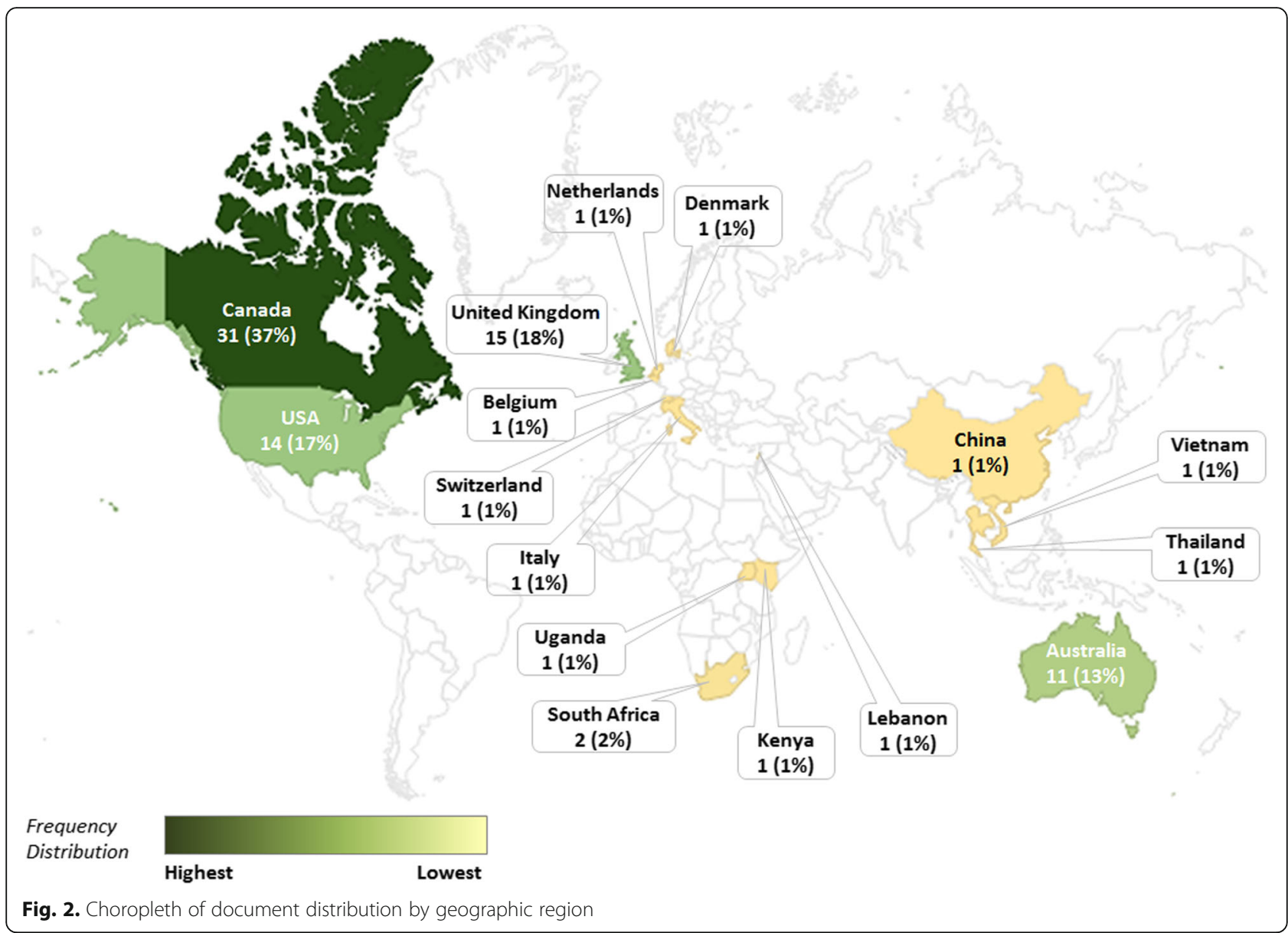

Full definitions for all terms can be found in Additional file 1: Appendix 3.

Frequently used methods of engagement were structured meetings or workshops and information gathering by means of surveys, focus groups or interviews across all four stages. Other methods of engagement included nominal group techniques or Delphi approaches to problem-solve and reach decisions in a group setting as well as circulating documents for feedback, and sending regular updates to relevant knowledge users. Knowledge users were most commonly engaged in-person or telephone across all four stages of a knowledge synthesis. Other forums for engagement included online platforms and email discussions.

The frequency of knowledge user engagement varied across the 73 application documents (Fig. 6). Knowledge users were engaged only once during the knowledge synthesis process in two-fifths of the documents, twice in onequarter of the documents, three times in one-tenth of the documents and in all four stages in nearly one-quarter of the documents.

\section{RQ2: Frameworks used to inform engagement strategy} One document reported the use of a framework for engagement in research [56] called the 7Ps of Stakeholder
Engagement and Six Stages of Research [57]. The 7Ps are (1) patients and the public, (2) providers, (3) purchasers, (4) payers, (5) policy-makers, (6) product makers, and (7) principal investigators. The six stages of research are (1) evidence prioritization, (2) evidence generation, (3) evidence synthesis, (4) evidence integration, (5) dissemination and application, and (6) feedback and assessment [57]. The authors of this framework recommended the following: prioritizing engagement through funding opportunities and other initiatives and adopting a common taxonomy when working with knowledge users, experimenting with different engagement strategies and evaluating them on an ongoing basis, and reporting outcomes and continuous quality improvement to iterate and implement changes when required.

Another document provided a conceptual framework on the models and mechanisms for engaging policy-makers in systematic reviews that focus on health policy and systems research [47]. Mechanisms that can be used to bolster engagement with policymakers included the following: finding ongoing funding so researchers can answer questions posed by policy-makers, providing capacity-building to researchers and policy-makers to support engagement, 
Table 2 Contextual factors

\begin{tabular}{|c|c|c|}
\hline \multicolumn{2}{|l|}{ Contextual factors $(n=84)$} & \multirow{2}{*}{$\frac{\text { Count (\%) }}{1(1.2 \%)}$} \\
\hline Settings & European Union Healthcare Systems & \\
\hline & Community Health & $2(2.4 \%)$ \\
\hline & National Public Health & $2(2.4 \%)$ \\
\hline & Local hospital & $2(2.4 \%)$ \\
\hline & Global health & $3(3.6 \%)$ \\
\hline & Health network & $4(4.8 \%)$ \\
\hline & Provincial/state healthcare system & $4(4.8 \%)$ \\
\hline & Various policy settings & $5(6.0 \%)$ \\
\hline & Local healthcare system & $5(6.0 \%)$ \\
\hline & Applied research setting & $16(19.0 \%)$ \\
\hline & National healthcare system & $40(47.6 \%)$ \\
\hline \multirow[t]{17}{*}{ Focus of knowledge synthesis } & Health economics & $1(1.2 \%)$ \\
\hline & Research reporting guideline & $1(1.2 \%)$ \\
\hline & Health informatics & $1(1.2 \%)$ \\
\hline & Emergency preparedness and management & $1(1.2 \%)$ \\
\hline & Community engagement & $1(1.2 \%)$ \\
\hline & Clinical practice guidelines & $3(3.6 \%)$ \\
\hline & Medical intervention & $3(3.6 \%)$ \\
\hline & Environmental/social determinants of health & $3(3.6 \%)$ \\
\hline & Quality indicators & $3(3.6 \%)$ \\
\hline & Health policy & $4(4.8 \%)$ \\
\hline & Decision-aid tool & $4(4.8 \%)$ \\
\hline & Research priority setting & $5(6.0 \%)$ \\
\hline & Health human resources & $8(9.5 \%)$ \\
\hline & Stakeholder engagement strategy in research & $9(10.7 \%)$ \\
\hline & Public health & $10(11.9 \%)$ \\
\hline & Knowledge translation & $13(15.5 \%)$ \\
\hline & Health services delivery & $14(16.7 \%)$ \\
\hline \multirow[t]{4}{*}{ Country economy } & High-income country & $72(85.7 \%)$ \\
\hline & Low- and middle-income country & $10(11.9 \%)$ \\
\hline & Middle and high income & $1(1.2 \%)$ \\
\hline & Low, middle, and high income & $1(1.2 \%)$ \\
\hline
\end{tabular}

and having team members with experience working closely with policy-makers.

\section{RQ3: Outcomes of engagement ( $n=84)$}

None of the included documents conducted a formal evaluation of engagement; measurement tools specific to engagement were not identified. The authors of one paper asked participating knowledge users to answer an anonymous survey and 100\% reported that the information provided in the review was "very" or "somewhat" useful in their decision-making [58]. One study [46] suggested ways to measure engagement in future research, including tracking how the research question, eligibility criteria, or other aspects of the review were modified after engagement, comparing reviews on the same topic with engagement and without engagement, retrospectively evaluating reviews that were conducted without engagement to determine their impact, or deliberately phasing in engagement at different parts of the process to measure how the engagement impacted the review.

RQ4: Barriers and facilitators to engagement $(n=31)$

Thirty-one documents reported on 16 factors that were considered barriers or facilitators to engagement (Table 6). The most common facilitators were content 


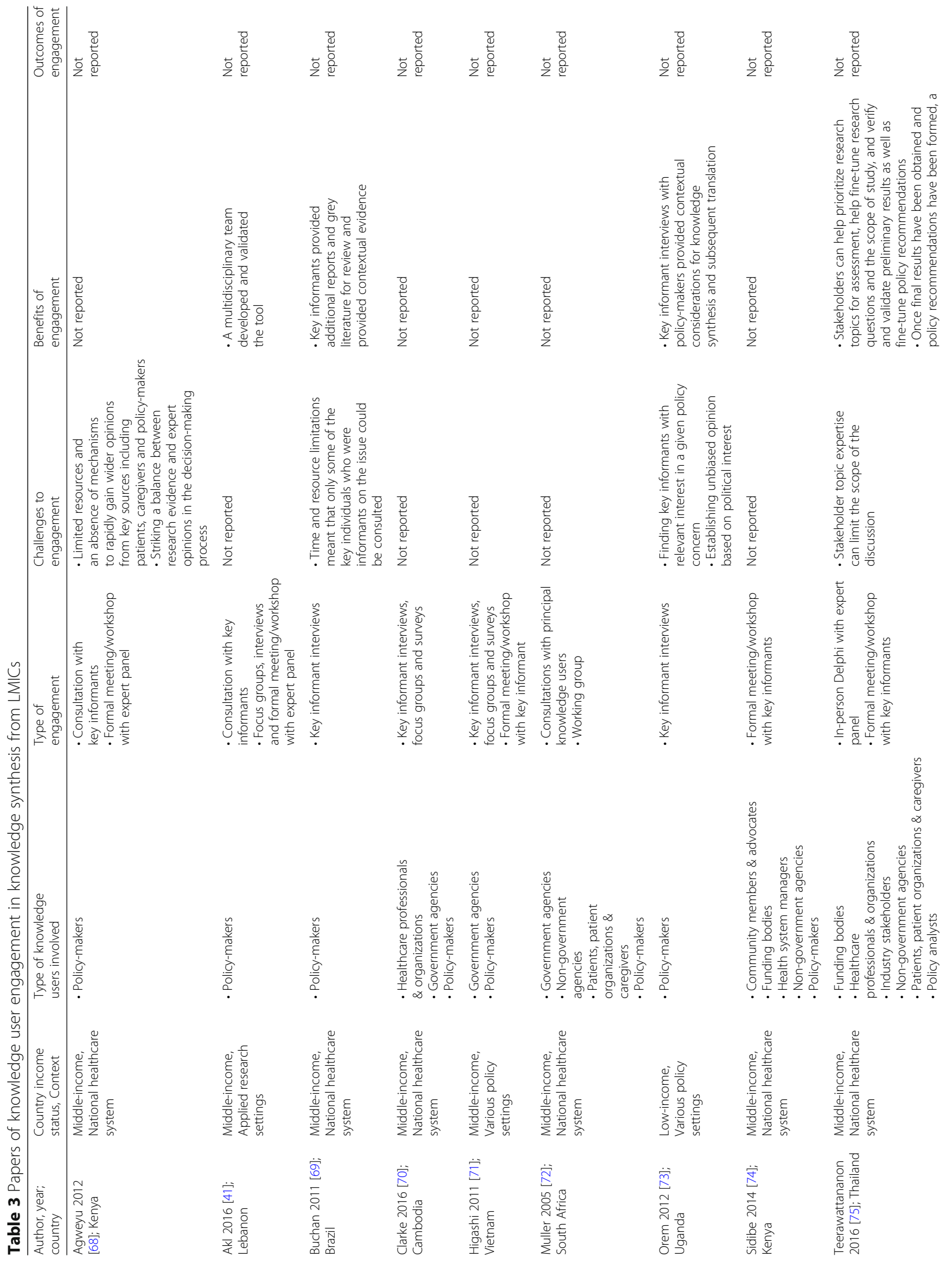




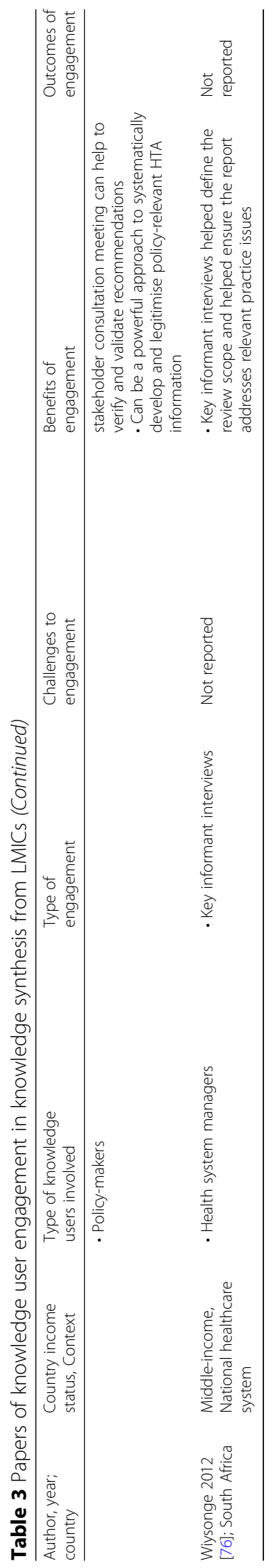


Table 4 Methodology papers of knowledge user engagement in knowledge synthesis

\begin{tabular}{|c|c|c|c|c|}
\hline $\begin{array}{l}\text { Article, Year; } \\
\text { Country }\end{array}$ & $\begin{array}{l}\text { Country income } \\
\text { status, Context }\end{array}$ & $\begin{array}{l}\text { Information } \\
\text { source }\end{array}$ & $\begin{array}{l}\text { Type of } \\
\text { engagement }\end{array}$ & $\begin{array}{l}\text { Challenges to } \\
\text { engagement }\end{array}$ \\
\hline $\begin{array}{l}\text { Cottrell } 2014 \\
\text { [46]; USA }\end{array}$ & $\begin{array}{l}\text { High-income, Applied } \\
\text { research settings }\end{array}$ & $\begin{array}{l}24 \text { articles, } 34 \\
\text { Key informant } \\
\text { interviews }\end{array}$ & Wide variety & $\begin{array}{l}\text { - Additional time and resources } \\
\text { - Selection of stakeholders/achieve } \\
\text { representativeness } \\
\text { - Reliability/consistency in participation } \\
\text { - Maintain confidentiality } \\
\text { - Manage and support stakeholders } \\
\text { Overcome tokenism }\end{array}$ \\
\hline $\begin{array}{l}\text { Guise } 2013 \\
\text { [48]; USA }\end{array}$ & $\begin{array}{l}\text { Low, middle and } \\
\text { high income, Applied } \\
\text { research settings }\end{array}$ & $\begin{array}{l}56 \text { articles, } 13 \\
\text { Key informant } \\
\text { interviews }\end{array}$ & $\begin{array}{l}\text { - One-on-one interviews } \\
\text { - Focus groups } \\
\text { - Citizen juries } \\
\text { - Town meetings } \\
\text { - Workshops/ } \\
\text { symposia/conferences } \\
\text { - Ranking and Delphi/ } \\
\text { Nominal group techniques }\end{array}$ & $\begin{array}{l}\text { - Lack of time on the part } \\
\text { of stakeholders (busy) } \\
\text { - Lack of release time and } \\
\text { compensation for members } \\
\text { of the public } \\
\text { - Researcher need for quick } \\
\text { response (time frame too } \\
\text { short for community to weigh in) } \\
\text { - Stakeholder needs not met in } \\
\text { previous engagement }\end{array}$ \\
\hline
\end{tabular}

Factors for successful engagements

- Engage stakeholders early in the process to establish credibility - Anticipate controversies in stakeholder opinions - Ensure transparency and accountability

- Engage stakeholders early in the process - Clearly detail expectations (e.g., timelines, tasks)

- Maintain ongoing relationships to building trust and credibility - Provide opportunities for people to ask questions before meetings - Provide pre-meeting information materials - Pre-meeting "icebreakers," especially when engaging stakeholders with differing experiences/ perspectives

- Include someone with similar training as the stakeholder can be helpful

- Respect and welcome all stakeholder opinions - Follow-up presentation of results is important to stakeholders - Be clear about the stakeholder roles, do not expect community members to do academic duties - Be sensitive to the time constraints of all stakeholders

Oliver 2016

[47]; UK

\begin{abstract}
High-income, Various policy settings
\end{abstract}

$\begin{array}{ll}18 \text { Key } & \cdot \text { Knowledge broker to } \\ \text { informant } & \text { facilitate conversations } \\ \text { interviews } & \begin{array}{l}\text { - Advisory panel and } \\ \text { Expert panel for } \\ \\ \end{array} \\ & \text { consultations }\end{array}$

$\begin{array}{ll}\text { - Lack of knowledge and } & \text { - Engage stakeholders } \\ \text { understanding between } & \text { early in the process } \\ \text { researchers and policy-makers } & \text { - Manage stakeholder } \\ \text { - Considerable time required } & \text { expectations } \\ \text { to negotiate review questions } & \text { - Maintain } \\ \text { with policy-makers } & \text { appropriate } \\ \text { - Researchers lacking experience } & \text { communication and } \\ \text { with stakeholder engagementldentifying } & \text { transparency } \\ \text { appropriate stakeholder to engage } & \text { - Face-to-face meetings } \\ \text { - Managing timelines, resources and } & \text { were more successful } \\ \text { costs associated with engagement } & \text { than telephone calls } \\ & \text { (not formally evaluated) }\end{array}$

- Lack of knowledge and understanding between researchers and policy-makers - Considerable time required with policy-makers appropriate stakeholder to engage costs associated with engagement expertise/awareness of the knowledge user (17\%), establishing partnership with knowledge users early in the research process (8\%), and having forums for ongoing interaction (7\%). The most common barriers reported were lack of time or opportunity for engagement (11\%) and when knowledge users lacked expertise/awareness of the topic (content) (6\%).

\section{Discussion}

The included documents were predominantly conducted at the level of a national healthcare system and focused on health services delivery in the context of highincome countries. We did not identify any distinguishing trends in engagement when we compared knowledge user engagement across country income groups 


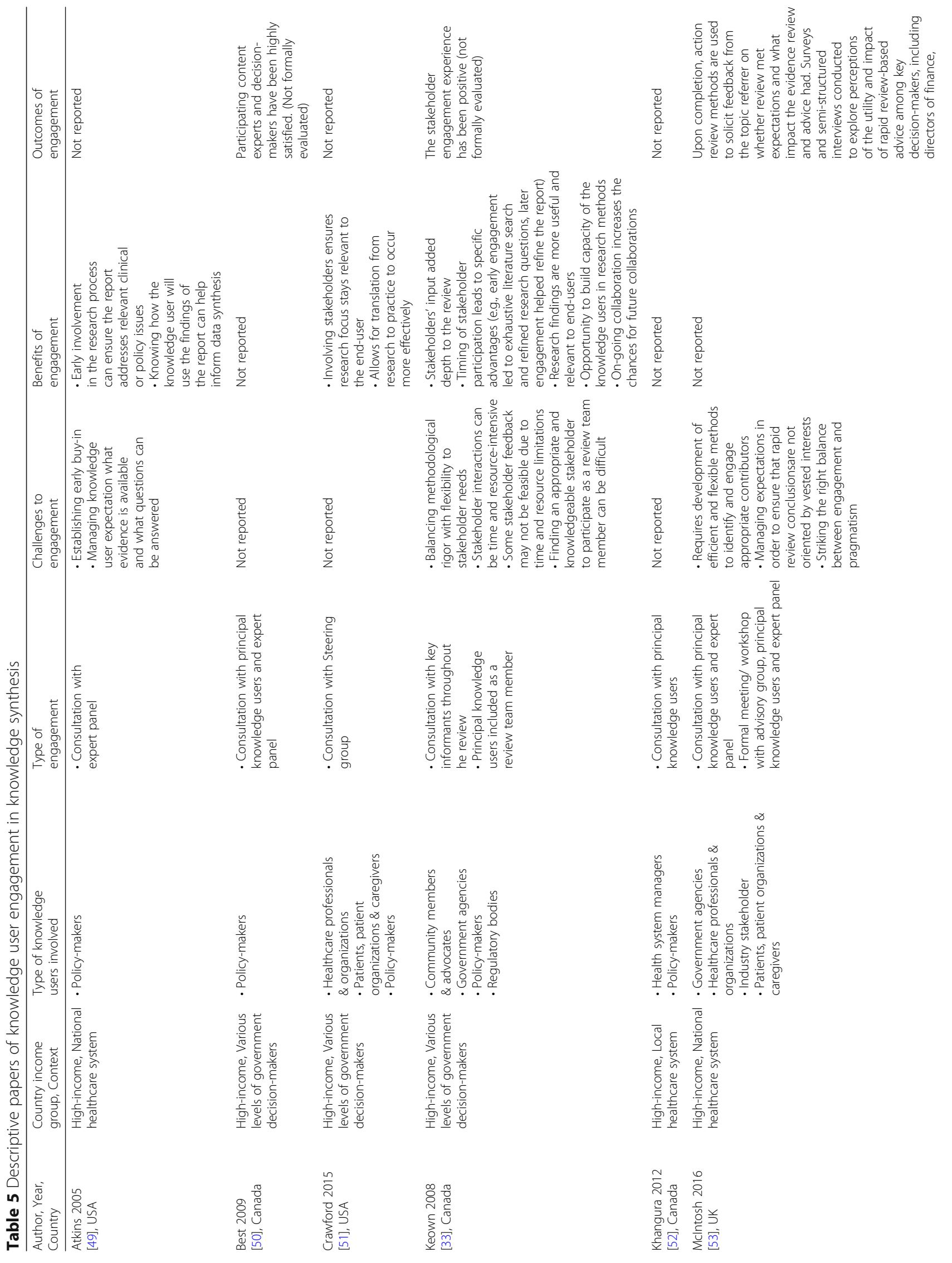




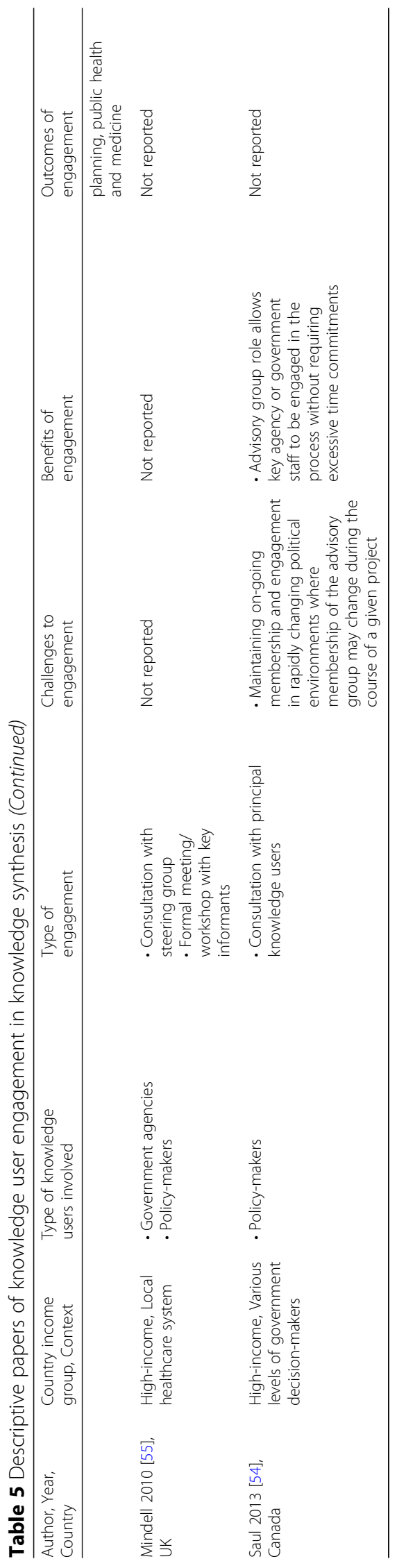




\begin{tabular}{|c|c|}
\hline \multicolumn{2}{|c|}{ 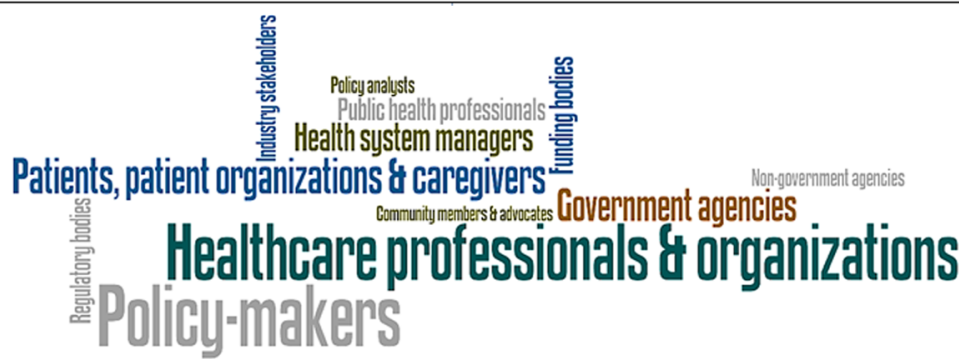 } \\
\hline Type of Knowledge Users* $(n=73)$ & Count (\%) \\
\hline Community members \& advocates & $3(4 \%)$ \\
\hline Policy analysts & $3(4 \%)$ \\
\hline Non-government agencies & $6(8 \%)$ \\
\hline Industry stakeholders & $8(11 \%)$ \\
\hline Regulatory bodies & $9(12 \%)$ \\
\hline Funding bodies & $10(14 \%)$ \\
\hline Public health professionals & $11(15 \%)$ \\
\hline Health system managers & $19(26 \%)$ \\
\hline Patients, patient organizations \& caregivers & $25(34 \%)$ \\
\hline Government agencies & $25(34 \%)$ \\
\hline Healthcare professionals \& organizations & $36(49 \%)$ \\
\hline Policy-makers & $47(64 \%)$ \\
\hline
\end{tabular}

*Note: One eligibility criterion for this review was that the article must mention engagement with either policymakers or health system level decision-makers. Each individual knowledge user can represent multiple categories.

Fig. 3. Types of knowledge users

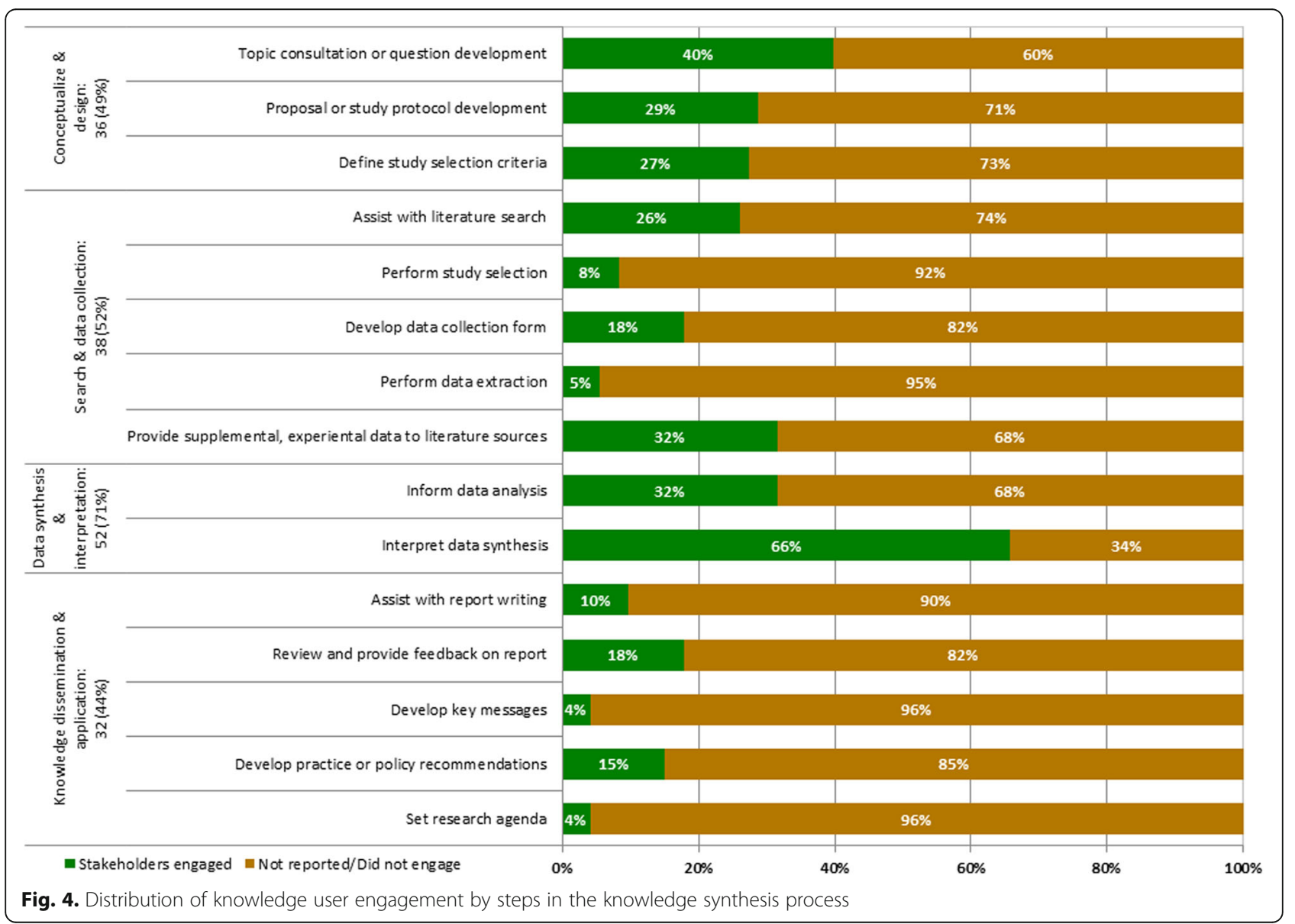




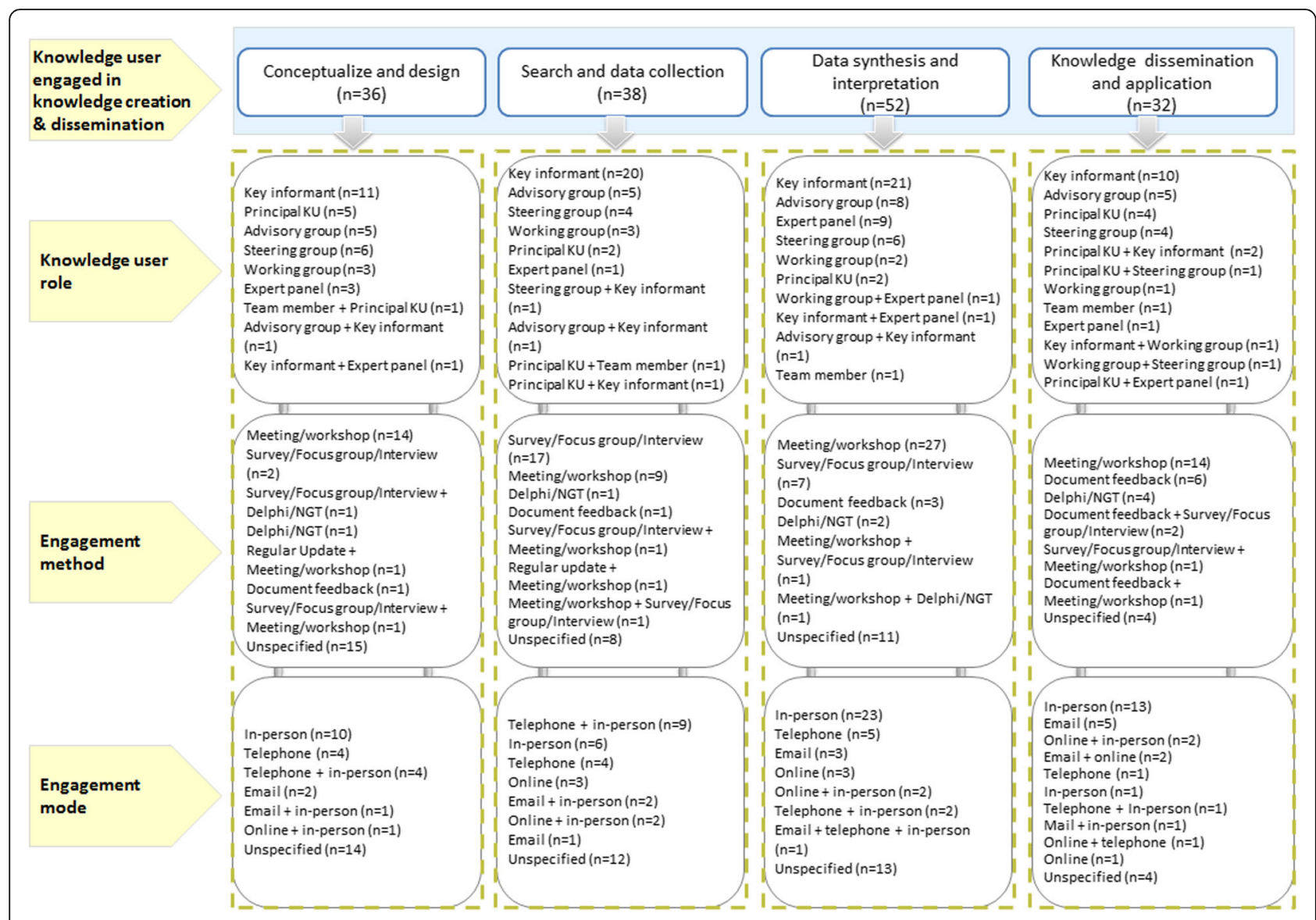

Fig. 5. Engagement strategy framework

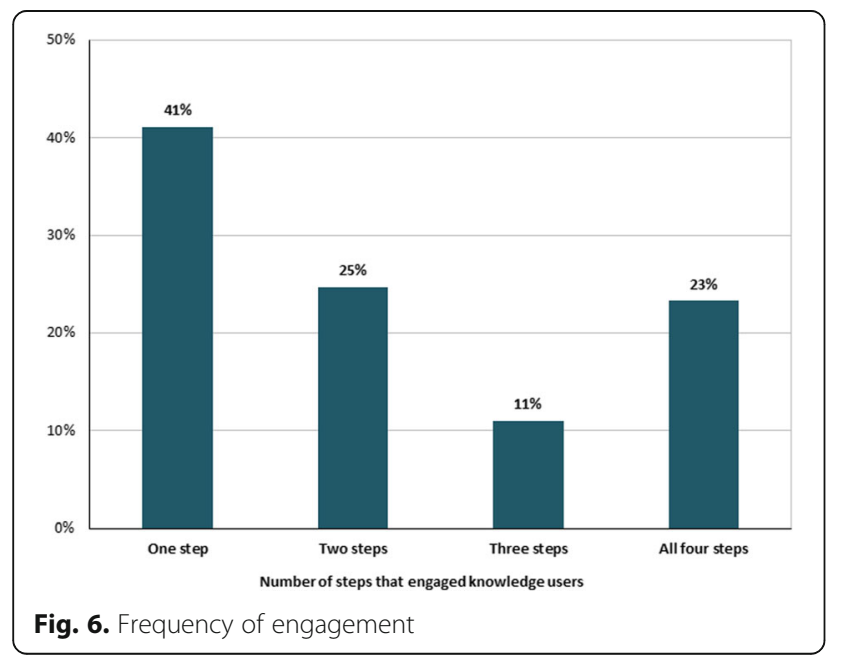

and other contextual factors. We did not identify differences in results over time or across settings, for phases of engagement, or how the engagement was conducted. This might be because the practice of engaging knowledge users in knowledge synthesis is still relatively new.

Knowledge users were most commonly engaged as key informants who were engaged through structured meetings or workshops and surveys, focus groups or interviews. Knowledge users were engaged only once during the knowledge synthesis process in two-fifths of the documents, twice in one-quarter of the documents, three times in one-tenth of the documents, and across all four stages in nearly one-quarter of the documents. None of the documents conducted a formal evaluation of engagement and measurement tools specific to engagement were not identified. Sixteen barriers and facilitators were identified. The most common facilitator was content expertise/awareness of the knowledge user, whereas the most common barrier was lack of time or opportunity for engagement.

There are numerous perceived benefits to engaging policy-makers, policy analysts, and health system managers in knowledge synthesis. Examples include more 
Table 6 Barriers and facilitators to engagement

\begin{tabular}{|c|c|c|}
\hline \multirow[t]{2}{*}{ Factors reported in 31 papers } & Seen as a facilitator & Seen as a barrier \\
\hline & Count (\%) & Count $(\%)$ \\
\hline Available resources (e.g., personnel, material) & $0(0.0 \%)$ & $2(2.4 \%)$ \\
\hline Capacity and established methods for engagement & $3(3.6 \%)$ & $1(1.2 \%)$ \\
\hline Clear expectations and responsibilities & $3(3.6 \%)$ & $1(1.2 \%)$ \\
\hline Contact with knowledge users & $2(2.4 \%)$ & $1(1.2 \%)$ \\
\hline Differing values & $1(1.2 \%)$ & $0(0.0 \%)$ \\
\hline $\begin{array}{l}\text { Establishment of partnership early in the research } \\
\text { process }\end{array}$ & $7(8.3 \%)$ & $0(0.0 \%)$ \\
\hline Establishment of unbiased consensus & $1(1.2 \%)$ & $2(2.4 \%)$ \\
\hline Forums for interaction & $6(7.1 \%)$ & $1(1.2 \%)$ \\
\hline Geographic distance & $0(0.0 \%)$ & $1(1.2 \%)$ \\
\hline Ongoing collaboration with partners & $4(4.8 \%)$ & $0(0.0 \%)$ \\
\hline Knowledge user research skills & $1(1.2 \%)$ & $1(1.2 \%)$ \\
\hline Knowledge user topic expertise/awareness & $14(16.7 \%)$ & $5(6.0 \%)$ \\
\hline Relationship with knowledge users & $2(2.4 \%)$ & $0(0.0 \%)$ \\
\hline Timing and opportunity & $3(3.6 \%)$ & $9(10.7 \%)$ \\
\hline $\begin{array}{l}\text { Training/mentoring of researchers and knowledge } \\
\text { users }\end{array}$ & $2(2.4 \%)$ & $0(0.0 \%)$ \\
\hline Willingness to participate & $1(1.2 \%)$ & $2(2.4 \%)$ \\
\hline
\end{tabular}

comprehensive literature searches, improved rigor of knowledge synthesis findings, greater clarity of results [59] as well as greater relevance, uptake, and usefulness of results. However, the results of our scoping review suggest that very little research has been conducted in this area. The research that has been conducted is purely descriptive in nature and a formal evaluation of engagement approaches and outcomes was not identified. A future study could evaluate engagement using a variety of methods, such as documenting how the knowledge synthesis process and results were modified after engagement or testing engagement at different points of the knowledge synthesis process to see how engagement influences research impact.

We identified several factors that may enhance engagement of knowledge users in knowledge synthesis process that are within the researcher's control, for example, engaging knowledge users before the synthesis begins; clearly outlining expectations regarding stakeholder's role and time commitment; identifying funding opportunities to work closely with policy-makers; providing time for question and answer opportunities; conducting ice breaker activities; providing materials in advance of meetings; considering knowledge user comments as being equal to those received from researchers; being sensitive to knowledge user's time; presenting results to knowledge users; and using a neutral facilitator. As none of these have been formally evaluated, we cannot comment on the effectiveness of any of these approaches. As such, the type and intensity of engagement should be meaningful and tailored to available resources, including time and funding. To better define knowledge user engagement in knowledge synthesis, researchers should discretely identify the desired benefits and impacts and effectiveness of engagement and develop systematic and reproducible methods and indicators for formal evaluation.

There were four main phases when engagement took place, including conception and design of research, search and data collection, data synthesis and interpretation, and knowledge dissemination and application.

Knowledge users were most often engaged as key informants across the four stages of the knowledge synthesis process to obtain advice, feedback, and opinions. However, there is increasing interest globally in co-design and co-development of research with knowledge users and using research to inform public policy $[60,61]$. Co-creation of science is gaining momentum to integrate research and decision-making cycles and incorporate knowledge generation in complex policy planning and implementation, ultimately enhancing the usability and impact of research [12, $62,63]$. It will be important to test the utility of the co-design and co-creation of knowledge synthesis in the future.

Two conceptual frameworks were identified that provided a structure and mechanism to facilitate knowledge user engagement in knowledge synthesis. These were the 7Ps of Stakeholder Engagement and Six Stages 
of Research framework [58] and a conceptual framework on the models and mechanisms for engaging policymakers in systematic reviews that focus on health policy and systems research [47]. An additional framework can also be used: the online survey patient and public engagement questionnaire (PPEQ) [64]. Members of our research team are currently conducting a study to test the level of engagement of knowledge users in a systematic review using the PPEQ [65], which will provide clarity to the field.

Two additional frameworks $[66,67]$ to engage stakeholders in knowledge synthesis were published after the literature search date and completion of our scoping review. Haddaway and colleagues [66] discussed a framework including approaches for engaging stakeholders during systematic reviews in the field of environmental management. Land and colleagues [67] described an empirically tested five-step approach for stakeholder engagement in prioritization and planning of environmental evidence syntheses that the Mistra Council for Evidence-based Environmental Management has been using. These frameworks may also be of relevance to knowledge synthesis within health and should be examined more closely in the future.

The strengths of our scoping review include a comprehensive literature search of multiple electronic databases as well as unpublished sources. We also followed the rigorous scoping review methods suggested by the Joanna Briggs Institute. We engaged with the principal knowledge user (EVL) throughout the review process who provided input in our research questions, review protocol, eligibility criteria of papers, reviewed this manuscript, and helped interpret our findings. In terms of dissemination plans, in addition to publication of this manuscript, we will prepare a1-page policy brief which will be made available on our website (https://knowledgetranslation.net/) and present at international conferences. Team members will also use their networks to encourage broad dissemination of results.

There are limitations to our scoping review process. To increase feasibility, we limited inclusion to documents made available in the past 20 years. However, this is likely not a substantial limitation, as all of the included documents were made available in the past 10 years. We also limited inclusion to studies written in English, which may have resulted in the exclusion of eligible studies from LMIC settings for RQ1. Given the large number of documents included, the data were abstracted by one reviewer and verified by a second reviewer. However, the data are likely valid, as a pilot-test was conducted prior to embarking on data abstraction with the entire team and a second reviewer who is an experienced research coordinator on the team verified all data. Often the included documents did not distinguish between stakeholders (i.e., those who are affected by or have an interest or stake in research [4]) and knowledge users (i.e., a subgroup of stakeholders who are likely to use research findings to make informed decisions about health systems and practices [5]), which is likely due to inconsistent use of the terms in the literature. As such, our results are likely applicable to both stakeholder and knowledge user participants. Furthermore, the reporting of knowledge user engagement methods varied considerably in their completeness across the literature, and as such, our data are limited by the details described in the literature. For example, most papers described steps to engage knowledge users but did not provide details on non-response or unsuccessful engagement.

\section{Conclusions}

Engaging policy-makers, policy analysts, and health system managers in knowledge synthesis usually occurs at the beginning or end of the knowledge synthesis process. However, ongoing engagement throughout the review process may lead to more relevant and user-friendly results. The type and intensity of engagement should be meaningful and tailored to available resources, including time and funding. Researchers should document and evaluate engagement activities in knowledge synthesis on an ongoing basis. It is important to document and test knowledge user engagement in knowledge synthesis in the future, to advance the field.

\section{Additional files}

Additional file 1: Appendices. The appendices includes all supplemental information. Appendix 1. Inclusion and exclusion criteria. Appendix 2. List of included papers. Appendix 3. Definitions. (DOCX 37 kb)

\section{Abbreviations}

HICs: High-income countries; LMICs: Low- and middle-income countries; PPEQ: Patient and public engagement questionnaire; PRISMA-P: Preferred reporting items for systematic reviews and meta-analysis for protocols; RQ: Research questions; WHO: World Health Organization

\section{Acknowledgements}

We thank Dr. Jessie McGowan for developing the search strategy and Dr. Elise Cogo for peer-reviewing it. We also thank Ms. Alissa Epworth for all library support, including performing the database and grey literature searches and obtaining full-text papers. Finally, we thank Ms. Theshani De Silva for formatting the manuscript and Ms. Susan Le for formatting the manuscript as well as providing all logistical and administrative support.

\section{Funding}

This study was funded by Alliance for Health Policy and Systems Research, WHO, Geneva, with support from the Norwegian Government Agency for Development Cooperation (Norad), the Swedish International Development Cooperation Agency (Sida) and the UK Department for International Development (DFID).

ACT is funded by a Tier 2 Canada Research Chair in Knowledge Synthesis, and SES is funded by a Tier 1 Canada Research Chair in Knowledge

Translation.

\section{Availability of data and materials}

The datasets used and/or analyzed during the current study are available from the corresponding author upon reasonable request. 


\section{Authors' contributions}

ACT obtained funding, conceptualized the research, participated in all pilottesting of screening and data abstraction forms, helped conceptualize the analysis, and wrote the first draft of the manuscript. WZ coordinated the study, screened citations and articles, resolved all discrepancies, cleaned the data, coded and verified the open-text data, performed all data analysis, created the figures, and drafted the sections of the manuscript. PR helped coordinate the study, screened the citations, abstracted the data, cleaned the data, coded the open-text data, and reviewed the manuscript. BP, MG, PK, SD, and VN helped with screening the citations and articles, abstracted and verified the data, and reviewed the manuscript. SES obtained funding, helped conceptualize the research, and reviewed the manuscript. EVL developed the research idea, helped conceptualized the study, and reviewed the manuscript. All authors read and approved the final manuscript.

\section{Ethics approval and consent to participate}

Since this is a scoping review, ethics approval was not required.

\section{Consent for publication}

Not applicable.

\section{Competing interests}

SES is an Associate Editor of the journal but was not involved with the submission process or decision to publish. All other authors declare that they have no competing interests.

\section{Publisher's Note}

Springer Nature remains neutral with regard to jurisdictional claims in published maps and institutional affiliations.

\section{Author details}

'Li Ka Shing Knowledge Institute of St. Michael's Hospital, 209 Victoria Street, Toronto, Ontario M5B 1T8, Canada. ${ }^{2}$ Epidemiology Division, Dalla Lana School of Public Health, University of Toronto, 6th Floor, 155 College St, Toronto, Ontario M5T 3M7, Canada. ${ }^{3}$ Department of Geriatric Medicine, Faculty of Medicine, University of Toronto, 27 King's College Circle, Toronto, Ontario M5S 1A1, Canada. ${ }^{4}$ Alliance for Health Policy and Systems Research World Health Organization, Avenue Appia 20, 1211 Geneva, Switzerland.

Received: 27 September 2017 Accepted: 25 January 2018

Published online: 12 February 2018

\section{References}

1. Chalmers I, Bracken MB, Djulbegovic B, Garattini S, Grant J, Gulmezoglu AM, et al. How to increase value and reduce waste when research priorities are set. Lancet. 2014;383(9912):156-65.

2. Chalmers I, Glasziou P. Avoidable waste in the production and reporting of research evidence. Lancet. 2009;374(9683):86-9.

3. Macleod MR, Michie S, Roberts I, Dirnagl U, Chalmers I, loannidis JP, et al. Biomedical research: increasing value, reducing waste. Lancet. 2014; 383(9912):101-4.

4. CIHR. Glossary of funding-related terms Canada: Canadian Institutes for Health Research; 2017. http://www.cihr-irsc.gc.ca/e/34190.html.

5. CIHR. Knowledge user engagement: Canadian Institutes of Health Research; 2016. http://www.cihr-irsc.gc.ca/e/49505.html].

6. CIHR. Integrated knowledge translation (iKT): Canadian Institutes of Health Research; 2015. http://www.cihr-irsc.gc.ca/e/45321.html\#a3.

7. Bragge P, Clavisi O, Turner T, Tavender E, Collie A, Gruen RL. The global evidence mapping initiative: scoping research in broad topic areas. BMC Med Res Methodol. 2011:11:92.

8. Deverka PA, Lavallee DC, Desai PJ, Esmail LC, Ramsey SD, Veenstra DL, et al. Stakeholder participation in comparative effectiveness research: defining a framework for effective engagement. J Comp Eff Res. 2012;1(2):181-94.

9. WHO. WHO strategy on health policy and systems research: changing mindsets. 2012. http://www.who.int/alliance-hpsr/alliancehpsr_ changingmindsets_strategyhpsr.pdf.

10. Bastian H, Glasziou P, Chalmers I. Seventy-five trials and eleven systematic reviews a day: how will we ever keep up? PLoS Med. 2010;7(9):e1000326.

11. Murad MH, Montori VM. Synthesizing evidence: shifting the focus from individual studies to the body of evidence. JAMA. 2013;309(21):2217-8.
12. Langlois EV, Becerril Montekio V, Young T, Song K, Alcalde-Rabanal J, Tran N Enhancing evidence informed policymaking in complex health systems: lessons from multi-site collaborative approaches. Health Res Policy Syst. 2016:14:20.

13. Vindrola-Padros C, Pape T, Utley M, Fulop NJ. The role of embedded research in quality improvement: a narrative review. BMJ Qual Saf. 2017; 26(1):70-80.

14. Ghaffar A, Langlois EV, Rasanathan K, Peterson S, Adedokun L, Tran NT. Strengthening health systems through embedded research. Bull World Health Organ. 2017;95(2):87.

15. Tricco AC, Zarin W, Rios P, Pham B, Straus SE, Langlois EV. Barriers, facilitators, strategies and outcomes to engaging policymakers, healthcare managers and policy analysts in knowledge synthesis: a scoping review protocol. BMJ Open. 2016;6(12):e013929.

16. Arksey H, O'Malley L. Scoping studies: towards a methodological framework. Int J Soc Res Methodol. 2005;8(1):19-32.

17. Tricco AC, Lillie E, Zarin W, O'Brien K, Colquhoun H, Kastner M, et al. A scoping review on the conduct and reporting of scoping reviews. BMC Med Res Methodol. 2016:16:15.

18. Peters M, Godfrey C, Mclnerney P, Soares C, Hanan K, Parker D. The Joanna Briggs Institute Reviewers' Manual 2015: methodology for JBI scoping reviews. 2015.

19. Shamseer L, Moher D, Clarke M, Ghersi D, Liberati A, Petticrew M, et al. Preferred reporting items for systematic review and meta-analysis protocols (PRISMA-P) 2015: elaboration and explanation. BMJ. 2015;349:97647.

20. Tricco AC, Zarin W, Rios P, Pham B, Straus SE, Langlois E. Barriers, facilitators, strategies and outcomes to engaging policy-makers, healthcare managers, and policy analysts in knowledge synthesis: a scoping review protocol. Open Science Framwork. 2016; https://osf.io/4dy53/

21. Stone PW. Popping the (PICO) question in research and evidence-based practice. Appl Nurs Res. 2002;15(3):197-8.

22. Langlois EV, Straus SE, Mijumbi-Deve R, Lewin S, Tricco AC. Chapter 1: the need for rapid reviews to inform health policy and systems. In: Tricco AC, Langlois EV, Straus SE, editors. Rapid reviews to strengthen health policy and systems: a. Practical Guide Geneva: World Health Organization; 2017.

23. McGowan J, Sampson M, Salzwedel DM, Cogo E, Foerster V, Lefebvre C. PRESS peer review of electronic search strategies: 2015 guideline statement. J Clin Epidemiol. 2016;75:40-6.

24. Grey Literature Report [Internet]. 2016. http://www.greylit.org/.

25. Open Grey [Internet]. 2017. http://www.opengrey.eu/.

26. EndNote. X7.7 ed: Thomson Reuters; 2016

27. Synthesi.SR [Internet]. Knowledge Translation Program, St. Michael's Hospital. 2012. http://www.breakthroughkt.ca/login.php.

28. The World Bank. World Bank Country and Lending Groups. https:// datahelpdesk.worldbank.org/knowledgebase/articles/906519.

29. Nilsen P. Making sense of implementation theories, models and frameworks. Implement Sci. 2015;10:53.

30. Tricco AC, Cardoso R, Thomas SM, Motiwala S, Sullivan S, Kealey MR, et al. Barriers and facilitators to uptake of systematic reviews by policy makers and health care managers: a scoping review. Implement Sci. 2016:11:4.

31. Gagliardi AR, Berta W, Kothari A, Boyko J, Urquhart R. Integrated knowledge translation (IKT) in health care: a scoping review. Implement Sci. 2016;11:38.

32. Oliver K, Innvar S, Lorenc T, Woodman J, Thomas J. A systematic review of barriers to and facilitators of the use of evidence by policymakers. BMC Health Serv Res. 2014;14(1):1-12.

33. Keown K, Van Eerd D, Irvin E. Stakeholder engagement opportunities in systematic reviews: knowledge transfer for policy and practice. J Contin Educ Heal Prof. 2008;28(2):67-72.

34. Carter N, Martin-Misener R, Kilpatrick K, Kaasalainen S, Donald F, BryantLukosius $\mathrm{D}$, et al. The role of nursing leadership in integrating clinical nurse specialists and nurse practitioners in healthcare delivery in Canada. Nurs Leadersh (Tor Ont). 2010;23 Spec No 2010:167-85.

35. Nieuwlaat R, Connolly SJ, Mackay JA, Weise-Kelly L, Navarro T, Wilczynski NL, et al. Computerized clinical decision support systems for therapeutic drug monitoring and dosing: a decision-maker-researcher partnership systematic review. Implement Sci. 2011;6:90.

36. Roshanov PS, You JJ, Dhaliwal J, Koff D, Mackay JA, Weise-Kelly L, et al. Can computerized clinical decision support systems improve practitioners' diagnostic test ordering behavior? A decision-maker-researcher partnership systematic review. Implement Sci. 2011;6:88.

37. Sahota N, Lloyd R, Ramakrishna A, Mackay JA, Prorok JC, Weise-Kelly L, et al. Computerized clinical decision support systems for acute care 
management: a decision-maker-researcher partnership systematic review of effects on process of care and patient outcomes. Implement Sci. 2011;6:91.

38. Souza NM, Sebaldt RJ, Mackay JA, Prorok JC, Weise-Kelly L, Navarro T, et al. Computerized clinical decision support systems for primary preventive care: a decision-maker-researcher partnership systematic review of effects on process of care and patient outcomes. Implement Sci. 2011;6:87.

39. Scotto Rosato N, Correll CU, Pappadopulos E, Chait A, Crystal S, Jensen PS. Treatment of maladaptive aggression in youth: CERT guidelines II. Treatments and ongoing management. Pediatrics. 2012;129(6):e1577-86.

40. Leonard MM, Agar M, Spiller JA, Davis B, Mohamad MM, Meagher DJ, et al. Delirium diagnostic and classification challenges in palliative care: subsyndromal delirium, comorbid delirium-dementia, and psychomotor subtypes. J Pain Symptom Manag. 2014;48(2):199-214.

41. Akl E, Fadlallah R, Ghandour L, Kdouh O, Langlois E, Lavis J, et al. The SPARK tool for prioritizing questions for systematic reviews in health policy and systems research: development and initial validation. Health Res Policy Syst. 15(1):77.

42. Hatziandreu E, Archontakis F, Daly A. The potential cost savings of greater use of home and hospice-based end of life care in England: RAND Corporation; 2008.

43. Committee on Integrating Primary Care and Public Health. Primary care and public health-exploring integration to improve population health 2012.

44. Pakes B. Ethical analysis in public health practice-Heterogeny, Discensus and the Man-on-the-Clapham Omnibus Toronto: University of Toronto; 2014.

45. Rycroft-Malone J, Burton CR, Williams L, Edwards S, Fisher D, Hall B, et al. Health services and delivery research. Improving skills and care standards in the support workforce for older people: a realist synthesis of workforce development interventions. Southampton: NIHR Journals Library; 2016.

46. Cottrell E, Whitlock E, Kato E, Uhl S, Belinson S, Chang C, et al. AHRQ methods for effective health care. Defining the benefits of stakeholder engagement in systematic reviews. Rockville: Agency for Healthcare Research and Quality (US); 2014.

47. Oliver S, Dickson K. Policy-relevant systematic reviews to strengthen health systems: models and mechanisms to support their production. Evidence \& Policy: J Res, Debate Pract. 2016;12(2):235-59.

48. Guise JM, O'Haire C, McPheeters M, Most C, Labrant L, Lee K, et al. A practice-based tool for engaging stakeholders in future research: a synthesis of current practices. J Clin Epidemiol. 2013;66(6):666-74.

49. Atkins D, Fink K, Slutsky J. Better information for better health care: the evidence-based practice center program and the Agency for Healthcare Research and Quality. Ann Intern Med. 2005;142(12 Pt 2):1035-41.

50. Best A, Terpstra JL, Moor G, Riley B, Norman CD, Glasgow RE. Building knowledge integration systems for evidence-informed decisions. J Health Organ Manag. 2009;23(6):627-41.

51. Crawford C, Boyd C, Jain S, Khorsan R, Jonas W. Rapid Evidence Assessment of the Literature $(\operatorname{REAL}((\mathrm{C})))$ : streamlining the systematic review process and creating utility for evidence-based health care. BMC Res Notes. 2015;8:631.

52. Khangura S, Konnyu K, Cushman R, Grimshaw J, Moher D. Evidence summaries: the evolution of a rapid review approach. Syst Rev. 2012;1:10.

53. McIntosh HM, Calvert J, Macpherson KJ, Thompson L. The healthcare improvement Scotland evidence note rapid review process: providing timely, reliable evidence to inform imperative decisions on healthcare. Int J Evid Based Healthc. 2016;14(2):95-101.

54. Saul JE, Willis CD, Bitz J, Best A. A time-responsive tool for informing policy making: rapid realist review. Implement Sci. 2013;8:103.

55. Mindell J, Bowen C, Herriot N, Findlay G, Atkinson S. Institutionalizing health impact assessment in London as a public health tool for increasing synergy between policies in other areas. Public Health. 2010;124(2):107-14.

56. Concannon TW, Fuster M, Saunders T, Patel K, Wong JB, Leslie LK, et al. A systematic review of stakeholder engagement in comparative effectiveness and patient-centered outcomes research. J Gen Intern Med. 2014;29(12):1692-701.

57. Concannon TW, Meissner P, Grunbaum JA, McElwee N, Guise JM, Santa J, et al. A new taxonomy for stakeholder engagement in patient-centered outcomes research. J Gen Intern Med. 2012;27(8):985-91.

58. Hayden JA, Killian L, Zygmunt A, Babineau J, Martin-Misener R, Jensen JL, et al. Methods of a multi-faceted rapid knowledge synthesis project to inform the implementation of a new health service model: collaborative emergency centres. Syst Rev. 2015;4:7.

59. Tricco AC, Langlois EV, Straus SE, editors. Rapid reviews to strengthen health policy and systems: a practical guide. Geneva: World Health Organization; 2017. Licence: CC BY-NC-SA 3.0 IGO

60. Goodyear-Smith F, Jackson C, Greenhalgh T. Co-design and implementation research: challenges and solutions for ethics committees. BMC Med Ethics. 2015;16:78.
61. Oliver K, Lorenc T, Innvaer S. New directions in evidence-based policy research: a critical analysis of the literature. Health Res Policy Syst. 2014;12:34.

62. Jackson CL, Greenhalgh T. Co-creation: a new approach to optimising research impact? Med J Aust. 2015:203(7):283-4.

63. Greenhalgh T, Jackson C, Shaw S, Janamian T. Achieving research impact through co-creation in community-based health services: literature review and case study. Milbank Q. 2016;94(2):392-429.

64. Moore A, Brouwers M, Straus SE, Tonelli M. Advancing patient and public involvement in guideline development. Toronto: Canadian Taskforce on Preventative Health Care; 2015.

65. Soobiah C, Daly C, Blondal E, Ewusie J, Ho J, Elliott MJ, et al. An evaluation of the comparative effectiveness of geriatrician-led comprehensive geriatric assessment for improving patient and healthcare system outcomes for older adults: a protocol for a systematic review and network meta-analysis. Syst Rev. 2017;6(1):65.

66. Haddaway N, Kohl C, da Silva NR, Schiemann J, Spök A, Stewart R, et al. A framework for stakeholder engagement during systematic reviews and maps in environmental management. Environmental Evidence. 2017;6(1):11.

67. Land M, Macura B, Bernes C, Johansson S. A five-step approach for stakeholder engagement in prioritisation and planning of environmental evidence syntheses. Environmental Evidence. 2017;6(1):25.

68. Agweyu A, Opiyo N, English M. Experience developing national evidencebased clinical guidelines for childhood pneumonia in a low-income setting — making the GRADE? BMC Pediatr. 2012;12:1.

69. Buchan J, Fronteira I, Dussault G. Continuity and change in human resources policies for health: lessons from Brazil. Hum Resour Health. 2011;9:17.

70. Clarke D, Duke J, Wuliji T, Smith A, Phuong K, San U. Strengthening health professions regulation in Cambodia: a rapid assessment. Hum Resour Health. 2016;14:9.

71. Higashi H, Khuong TA, Ngo AD, Hill PS. The development of Tobacco Harm Prevention Law in Vietnam: stakeholder tensions over tobacco control legislation in a state owned industry. Subst Abuse Treat Prev Policy. 2011;6:24.

72. Muller L, Flisher A. Standards for the mental health care of people with severe psychiatric disorders in South Africa: part 2. Methodology and results. South African Psychiatr Rev. 2005;8:146-52.

73. Orem JN, Mafigiri DK, Marchal B, Ssengooba F, Macq J, Criel B. Research, evidence and policymaking: the perspectives of policy actors on improving uptake of evidence in health policy development and implementation in Uganda. BMC Public Health. 2012:12:109.

74. Sidibe S, Pack AP, Tolley EE, Ryan E, Mackenzie C, Bockh E, et al. Communicating about microbicides with women in mind: tailoring messages for specific audiences. J Int AIDS Soc. 2014;17(3 Suppl 2):19151.

75. Teerawattananon Y, Kingkaew P, Koopitakkajorn T, Youngkong S, Tritasavit $N$, Srisuwan $P$, et al. Development of a health screening package under the universal health coverage: the role of health technology assessment. Health Econ. 2016;25(Suppl 1):162-78.

76. Wiysonge CS, Ngcobo NJ, Jeena PM, Madhi SA, Schoub BD, Hawkridge A, et al. Advances in childhood immunisation in South Africa: where to now? Programme managers' views and evidence from systematic reviews. BMC Public Health. 2012:12:578

\section{Submit your next manuscript to BioMed Central and we will help you at every step:}

- We accept pre-submission inquiries

- Our selector tool helps you to find the most relevant journal

- We provide round the clock customer support

- Convenient online submission

- Thorough peer review

- Inclusion in PubMed and all major indexing services

- Maximum visibility for your research

Submit your manuscript at www.biomedcentral.com/submit
Biomed Central 